\title{
ANÁLISE DA QUALIDADE DE VIDA E SINTOMAS DEPRESSIVOS EM IDOSOS COM QUEIXA DE TONTURA
}

\author{
Camila Rosseto Ludwig \\ Pricila Sleifer ${ }^{2}$ \\ Amanda Zanatta Berticelli ${ }^{3}$ \\ Audrei Thayse Viegel de Ávila ${ }^{4}$
}

resumo

Objetivo: analisar a qualidade de vida e a presença de sintomas depressivos em idosos com queixa de tontura. Método: estudo transversal, individual, observacional e contemporâneo. Foram analisadas as respostas da Anamnese, do Questionário SF-36 e da Escala de Depressão Geriátrica (GDS) de 153 idosos com queixa de tontura. Resultados: 64,1\% dos idosos pontuaram acima do ponto de corte

1 Graduada em Fonoaudiologia. Especialista em Audiologia (UFRGS). E-mail: camila.fono@yahoo. com.br

2 Graduada em Fonoaudiologia. Doutora em Ciências Médicas pela UFRGS. Professora Adjunta IV do Departamento de Saúde e Comunicação Humana (UFRGS). E-mail: pricilasleifer@uol.com.br 3 Graduada em Fonoaudiologia. Fonoaudióloga do Hospital São Lucas da PUCRS. E-mail: azanattab@hotmail.com

4 Graduada em Fonoaudiologia. Fonoaudióloga residente multiprofissional: Atenção Básica em Saúde Coletiva pela Escola de Saúde Pública do Estado do Rio Grande do Sul. E-mail: audreiviegel@ hotmail.com 
na Escala de Depressão Geriátrica, sendo que os percentuais foram semelhantes entre homens e mulheres ( $p=0,723)$. Houve correlação inversa entre a pontuação na GDS e todos os domínios do SF-36, ou seja, quanto maior a pontuação na GDS, menor a qualidade de vida em todos os domínios. A frequência da tontura correlacionou-se inversamente com os domínios do SF-36, exceto no domínio dor, isto é, quanto mais frequente a tontura, menor a qualidade de vida. Houve correlação direta entre a pontuação na GDS com a idade e com a frequência da tontura: quanto maior a idade e a frequência da tontura, maior o grau de depressão ( $p<0,001)$. Conclusão: idosos com queixa de tontura apresentam prejuízos significativos na qualidade de vida. A prevalência de sintomas depressivos foi significativa na amostra estudada. Houve associação entre a presença de sintomas depressivos e prejuízos na qualidade de vida. Estes achados corroboram com dados prévios da literatura, que destacam os prejuízos da tontura na qualidade de vida de idosos, bem como a presença de sintomas depressivos concomitantes a essa queixa nesses sujeitos, chamando atenção para a necessidade de uma abordagem multidisciplinar no manejo terapêutico desses pacientes.

palavras - chave

Tontura. Idoso. Qualidade de Vida. Depressão.

As alterações do equilíbrio corporal, que se caracterizam clinicamente como tontura, vertigem, desequilíbrio e queda, estão entre as queixas mais comuns da população geriátrica (SIMOCELI et al., 2003; FELIPE et al., 2008; MANTELLO et al., 2008). O envelhecimento afeta a habilidade do sistema nervoso central de processar os sinais vestibulares, visuais e proprioceptivos que desempenham papel fundamental na manutenção do equilíbrio corporal (RUWER; ROSSI; SIMON, 2005).

A tontura pode ser vista como uma síndrome geriátrica, de origem multifatorial decorrente do efeito cumulativo de alterações em diversos sistemas, provocando nos indivíduos idosos dificuldades em lidar com a variabilidade dos contextos ambientais e atividades do cotidiano (TINETTI; WILLIAMS; GILL, 2000; BITTAR; GANANÇA, 2010). Além disso, pode interferir de maneira significativa na qualidade de vida, gerando incapacitação parcial ou total no 
desempenho de atividades sociais e profissionais (GANANÇA; CAOVILLA, 1998; GURGEL et al., 2012).

A qualidade de vida tem sido um tema amplamente discutido e uma preocupação constante das pessoas (BITTAR; GANANÇA, 2010). A Organização Mundial de Saúde (WHO, 1980) definiu qualidade de vida como a percepção do indivíduo de sua posição na vida, no contexto de sua cultura e sistema de valores nos quais ele está inserido e em relação aos seus objetivos, expectativas, padrões e preocupações.

O Questionário SF-36, Medical Outcome Study36 - Item Short Form Health Survey - SF-36 (WARE et al., 1993), traduzido e adaptado para a população brasileira por Ciconelli (1997), tem sido amplamente utilizado para avaliar o impacto da doença na qualidade de vida (CAMPOLINA; CICONELLI, 2008). Trata-se de um instrumento versátil, curto e aplicável tanto por entrevista como por autoadministração.

Ao analisar a qualidade de vida de idosos com tontura por meio do questionário SF-36, Hsu et al. (2005) verificaram que, em comparação a dados normativos, os resultados dos pacientes com tontura indicaram queda generalizada multidimensional. Essa diminuição mostrou-se mais notável em termos de limitações nos aspectos físico e emocional e evidenciou que idosos com tontura crônica apresentam grande sofrimento psíquico, influenciando negativamente a qualidade de vida dos mesmos. A tontura está habitualmente acompanhada por sintomas de natureza psíquica nos indivíduos que a apresentam, podendo ser primária à tontura ou ocorrer como consequência desta, por vezes contribuindo com a piora do quadro clínico (BITTAR; GANANÇA, 2010). Indivíduos com tontura relatam com frequência dificuldade de concentração, perda de memória, irritabilidade, insônia ou sonolência e fadiga (IÓRIO, 2011). Além disso, a insegurança física provocada pelo distúrbio do equilíbrio corporal frequentemente conduz à insegurança psíquica, ansiedade, depressão e pânico (GANANÇA; CAOVILLA, 1998).

Estudos que investigaram a relação entre a tontura e distúrbios psíquicos sugerem que um elevado número de pacientes encontrou diminuição significativa dos sintomas depressivos após o manejo da tontura (BITTAR; GANANÇA, 2010; HONG et al., 2012). Sabendo-se que na população idosa a tontura é uma queixa frequente (GURGEL et al., 2012), habitualmente acompanhada por sintomas depressivos e com significativo impacto na qualidade de vida, faz-se necessário a investigação de tais condições patológicas para o adequado manejo terapêutico e avaliação desses pacientes.

O presente estudo tem o objetivo de analisar a qualidade de vida e a presença de sintomas depressivos em idosos com queixa de tontura, bem como 
correlacionar os achados com idade, gênero, grau de depressão, intensidade e frequência da tontura.

\section{Metodologia}

Este é um estudo transversal, individual, observacional e contemporâneo, no qual o fator em estudo foi idosos com queixa de tontura, e o desfecho clínico, a análise da qualidade de vida e a ocorrência de sintomas depressivos.

A casuística foi constituída de idosos provenientes de unidades de saúde das cidades de Porto Alegre e Carazinho, RS. Optou-se em realizar as coletas nesses locais para a obtenção de um maior número de participantes. Os dados foram coletados no período de julho a novembro de 2011. Para estimar um tamanho de efeito padronizado de 0,7 (moderado), foi calculado um tamanho amostral mínimo de 102 idosos, considerando o nível de significância de 0,05 com poder de $90 \%$ (EpiInfo 6 - Statcal).

Esta pesquisa foi aprovada pelo Comitê de Ética em Pesquisa do Instituto de Psicologia da UFRGS, sob o protocolo número 2011040. Os participantes foram devidamente esclarecidos sobre os propósitos da pesquisa e concordaram com o Termo de Consentimento Livre e Esclarecido. Foram incluídos indivíduos que apresentaram queixa de tontura e idade igual ou superior a 60 anos (de acordo com a Portaria no 1.395/GM da Política Nacional do Idoso; BRASIL, 1999). Foram excluídos do estudo os participantes que apresentaram perdas cognitivas, rastreados pelo mini exame de estado mental, de acordo com Brucki et al. (2003), histórico prévio de lesão neurológica, ou que se negaram a participar do estudo.

Primeiramente, realizou-se a anamnese. As perguntas foram feitas pelos pesquisadores e compreenderam questões ligadas a dados pessoais, dados sociodemográficos, queixa principal e suas características, sintomas associados e suas características e antecedentes patológicos e familiares. Na anamnese, a intensidade da tontura foi autorrelatada pelos idosos, baseada na sua percepção subjetiva. Após a anamnese, os pesquisadores aplicaram o questionário do mini exame do estado mental, originalmente proposto por Folstein, Folstein e McHugh (1975) e padronizado para a população brasileira por Bertolucci et al. (1994). Trata-se de um instrumento clínico utilizado na detecção de perdas cognitivas, que avalia orientação, memória imediata, atenção, cálculo, evocação e linguagem. O resultado é analisado em função da escolaridade, sendo o ponto de corte, de acordo com a classificação de Brucki et al. (2003), 
de 20 pontos para analfabetos, 25 para escolaridade entre 1 e 4 anos, 26,5 para escolaridade entre 5 a 8 anos, 28 para escolaridade entre 9 e 11 anos e 29 para escolaridade superior a 11 anos. Dessa forma, foram excluídos do estudo aqueles que apresentaram perdas cognitivas.

Posteriormente, foram aplicados respectivamente o Questionário Genérico de Qualidade de Vida SF-36 (CICONELLI, 1997) e o questionário pertencente à Escala de Depressão Geriátrica Breve (GDS), versão abreviada com 15 perguntas (YESAVAGE; SHEIK, 1986). O SF-36 (Medical Outcomes Study 36 -Item Short-Form Health Survey) é um instrumento genérico de avaliação de qualidade de vida. Trata-se de um questionário multidimensional, composto por 36 itens que contemplam oito escalas ou componentes: capacidade funcional, aspectos físicos, dor, estado geral de saúde, vitalidade, aspectos emocionais, aspectos sociais e saúde metal. Possui um escore final de zero a 100, sendo que zero é considerado como o pior estado de saúde e 100, o melhor estado de saúde (CICONELLI, 1997).

A GDS é uma escala amplamente utilizada para rastrear sintomas depressivos em indivíduos idosos. É composta de 15 questões com respostas do tipo "Sim" ou "Não", necessitando de um curto tempo para sua aplicação (YESAVAGE; SHEIK, 1986; FIGUEIREDO et al., 2003). O ponto de corte para este estudo foi de cinco pontos, sendo a classificação:

- Menos de 05 pontos: ausência de sintomatologia depressiva;

- Entre 05 e 10 pontos: sintomatologia depressiva leve a moderada;

- Mais de 10 pontos: sintomatologia depressiva grave.

Os questionários foram aplicados oralmente pelos próprios pesquisadores em um único dia, com duração de cerca de 30 minutos. Além do fator em estudo principal e do desfecho, foram consideradas as variáveis: gênero, idade e frequência da tontura. A análise dos dados foi realizada utilizando o software SPSS (Statistical Package for the Social Sciences), versão 17.0. As variáveis quantitativas foram descritas por meio de média e desvio padrão ou mediana e amplitude de variação. As variáveis categóricas foram descritas por meio de frequências absolutas e relativas. Para avaliar a correlação entre as variáveis contínuas e ordinais, o coeficiente de correlação de Spearman foi aplicado. A correlação entre as variáveis categóricas foi avaliada pelo teste qui-quadrado de Pearson. Para comparar o escore de qualidade de vida entre os gêneros, foram aplicados os testes de Mann-Whitney ou Kruskal-Wallis. O nível de significância estatística considerado foi de $5 \%(p \leq 0,05)$. 
A casuística deste estudo foi constituída de 153 indivíduos idosos que apresentavam queixa de tontura. A média de idade foi de 72,5 anos $( \pm 7,7)$, sendo a mínima 60 e a máxima, 91 anos.

Os dados sociodemográficos, a intensidade e a frequência da tontura estão descritos na Tabela 1.

Tabela 1 - Caracterização da amostra.

\begin{tabular}{|c|c|}
\hline Variáveis* & Total $(n=153)$ \\
\hline Idade (anos) & $72,5 \pm 7,7$ \\
\hline $60-69$ & $62(40,5)$ \\
\hline $70-79$ & $55(35,9)$ \\
\hline$\geq 80$ & $36(23,5)$ \\
\hline \multicolumn{2}{|l|}{ Gênero } \\
\hline Masculino & $46(30,1)$ \\
\hline Feminino & $107(69,9)$ \\
\hline \multicolumn{2}{|l|}{ Estado civil } \\
\hline Solteiro & $15(9,8)$ \\
\hline Casado & $80(52,3)$ \\
\hline Viúvo & $52(34,0)$ \\
\hline Divorciado & $6(3,9)$ \\
\hline \multicolumn{2}{|c|}{ Intensidade da tontura (percepção subjetiva do indivíduo) } \\
\hline Leve & $59(38,6)$ \\
\hline Moderada & $52(34,0)$ \\
\hline Intensa & $41(26,8)$ \\
\hline Exacerbada & $1(0,7)$ \\
\hline \multicolumn{2}{|c|}{ Frequência da tontura } \\
\hline Esporádica (mensal) & $95(62,1)$ \\
\hline Frequente (semanal) & $50(32,7)$ \\
\hline Muito frequente (diária) & $8(5,2)$ \\
\hline
\end{tabular}

* variáveis descritas por média \pm desvio padrão ou n(\%) 
Em relação às respostas ao questionário SF-36 brasileiro, as médias dos domínios encontram-se na Tabela 2. Na Tabela 3, encontram-se as medianas dos domínios de acordo com o gênero. Não houve diferença significativa entre homens e mulheres quanto à qualidade de vida.

Tabela 2 - Avaliação da qualidade de vida por meio do questionário SF-36

\begin{tabular}{l|c|c}
\hline \multicolumn{1}{c|}{ Domínios } & Média \pm DP & Mediana (mín.-máx.) \\
\hline Capacidade funcional & $52,6 \pm 24,3$ & $55(30-70)$ \\
\hline Limitação por aspectos físicos & $20,8 \pm 27,3$ & $0(0-25)$ \\
\hline Dor & $44,8 \pm 22,3$ & $41(20-61)$ \\
\hline Estado geral de saúde & $50,1 \pm 19,8$ & $47(35-67)$ \\
\hline Vitalidade & $49,8 \pm 18,1$ & $50(35-65)$ \\
\hline Aspectos sociais & $58,6 \pm 27,2$ & $50(37,5-75)$ \\
\hline Limitação por aspectos emocionais & $39,4 \pm 32,5$ & $33,3(25-66,7)$ \\
\hline Saúde mental & $55,0 \pm 20,9$ & $56(36-70)$ \\
\hline
\end{tabular}

Questionário SF-36 = Medical Outcome Study36 - Item Short Form Health Survey - SF-36 $\mathrm{DP}$ = desvio padrão; Mín. = mínimo; Máx = máximo.

Tabela 3 - Avaliação da qualidade de vida por meio do SF-36 conforme gênero

\begin{tabular}{l|c|c|c}
\hline \multicolumn{1}{c|}{ Domínios } & $\begin{array}{c}\text { Homens } \\
\text { Mediana (mín.-máx.) }\end{array}$ & $\begin{array}{c}\text { Mulheres } \\
\text { Mediana (mín.-máx.) }\end{array}$ & Valor $\boldsymbol{p}$ \\
\hline Capacidade funcional & $60(40-70)$ & $50(30-70)$ & 0,219 \\
\hline Limitação por aspectos físicos & $25(0-25)$ & $0(0-25)$ & 0,519 \\
\hline Dor & $41(31-52)$ & $41(20-61)$ & 0,986 \\
\hline Estado geral de saúde & $45(40-67)$ & $47(35-67)$ & 0,864 \\
\hline Vitalidade & $50(35-65)$ & $50(35-60)$ & 0,803 \\
\hline Aspectos sociais & $62,5(50-75)$ & $50(37,5-75)$ & 0,373 \\
\hline Limitação por aspectos & $33,3(25-66,7)$ & $33,3(33,3-66,7)$ & 0,849 \\
\hline emocionais & $60(50-69)$ & $52(36-72)$ & 0,239 \\
\hline Saúde mental & 540 (36-1 &
\end{tabular}

Questionário SF-36 = Medical Outcome Study 36 - Item Short Form Health Survey - SF-36 Min = mínimo; Max = máximo. P-valor referente ao teste de Mann-Whitney para comparação entre gêneros, com nível de significância estatística de 5\% (p<0,05). 
$\mathrm{Na}$ avaliação da presença de sintomas depressivos por meio da GDS, a amostra total evidenciou que 55 idosos (35,9\%) apresentaram ausência de sintomas depressivos e $98(64,1 \%)$ tiveram os sintomas presentes. Os percentuais foram semelhantes em homens e mulheres $(p=0,723)$, conforme pode ser visualizado na Figura 1.

Figura 1 - Avaliação da presença de sintomas depressivos, por meio da GDS, na amostra total e conforme o gênero.

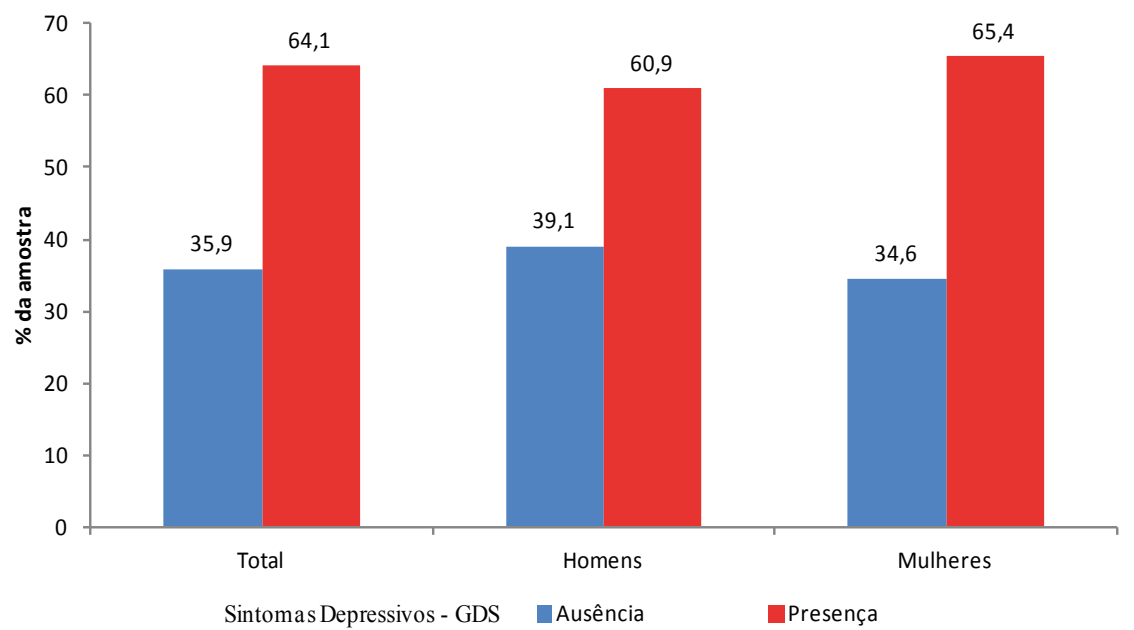

Teste qui-quadrado de Pearson. Nível de significância estatística de 5\% ( $p \leq 0,05)$. GDS = Escala de Depressão Geriátrica Breve.

Considerando o grau da depressão, 78 (51\%) idosos apresentaram pontuação sugestiva de depressão leve à moderada e 20 (13,1\%), sugestão de depressão grave. Os percentuais foram semelhantes em homens e mulheres $(p=0,676)$, como pode ser visualizado na Figura 2. 
Figura 2 - Avaliação do grau de depressão, através da GDS, na amostra total e conforme o gênero.

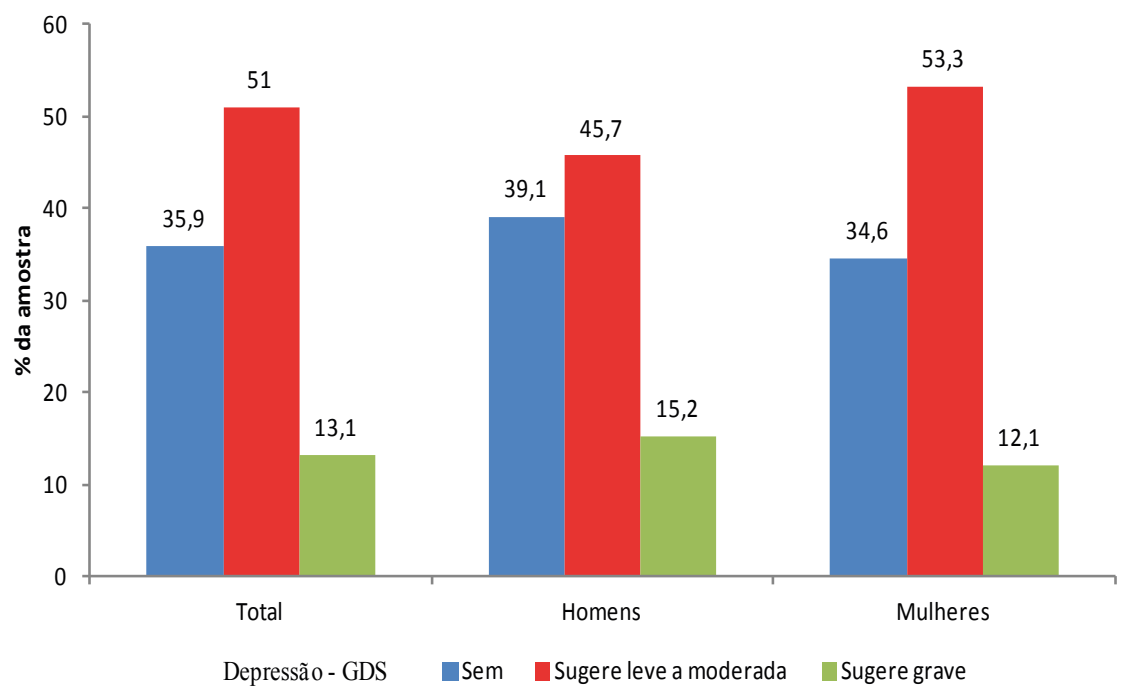

Teste qui-quadrado de Pearson. Nível de significância estatística de 5\% ( $p \leq 0,05)$. GDS = Escala de Depressão Geriátrica Breve.

Houve correlação inversa entre o grau de depressão e todos os domínios do SF-36, ou seja, quanto maior a pontuação na GDS, menor a qualidade de vida em todos os domínios, como pode ser visualizado na Tabela 4 . Também se observou uma associação inversa entre a idade com o domínio de vitalidade do SF-36, ou seja, quanto maior a idade, menor a qualidade de vida nesse domínio. 
Tabela 4 - Correlação da qualidade de vida com a idade, intensidade e frequência da tontura e grau de depressão.

\begin{tabular}{|c|c|c|c|c|}
\hline Domínios & Idade & $\begin{array}{l}\text { Intensidade } \\
\text { da tontura }\end{array}$ & $\begin{array}{l}\text { Frequência } \\
\text { da tontura }\end{array}$ & $\begin{array}{l}\text { Grau de } \\
\text { depressão }\end{array}$ \\
\hline Capacidade funcional & $-0,104$ & $-0,081$ & $-0,328^{\star \star \star}$ & $-0,355^{\star \star \star}$ \\
\hline Limitação por aspectos físicos & $-0,136$ & $-0,045$ & $-0,190^{*}$ & $-0,290^{\star \star \star}$ \\
\hline Dor & $-0,099$ & 0,042 & $-0,149$ & $-0,389^{\star \star \star}$ \\
\hline Estado geral de saúde & $-0,037$ & 0,010 & $-0,353^{\star \star \star}$ & $-0,465^{\star \star \star}$ \\
\hline Vitalidade & $-0,204^{*}$ & $-0,021$ & $-0,351^{\star \star \star}$ & $-0,587^{\star \star \star}$ \\
\hline Aspectos sociais & $-0,139$ & $-0,030$ & $-0,302^{\star \star \star}$ & $-0,561^{\star \star \star}$ \\
\hline $\begin{array}{l}\text { Limitação por aspectos } \\
\text { emocionais }\end{array}$ & $-0,103$ & $-0,117$ & $-0,472^{\star \star \star}$ & $-0,358^{\star \star \star}$ \\
\hline Saúde mental & $-0,125$ & $-0,112$ & $-0,247^{\star \star}$ & $-0,568^{\star \star \star}$ \\
\hline
\end{tabular}

Valores dos coeficientes de correlação de Spearman ${ }^{*} p<0,05 ;{ }^{* \star} p<0,01 ;{ }^{* \star *} p<0,001$

Além disso, a frequência da tontura associou-se inversamente com quase todos os domínios do SF-36, exceto de dor, ou seja, quanto mais frequente a tontura, menor a qualidade de vida.

Houve associação direta entre o grau de depressão com a idade e com a frequência de tontura, como pode ser visualizado na Tabela 5, ou seja, quanto maior a idade e a frequência da tontura, maior o grau de depressão.

Tabela 5 - Associação do grau de depressão com a idade e intensidade e ocorrência da tontura

\begin{tabular}{l|c|c}
\hline \multirow{2}{*}{ Variáveis } & \multicolumn{2}{|c}{ Grau de depressão } \\
\cline { 2 - 3 } & rs & Valor $\boldsymbol{p}$ \\
\hline Idade & 0,159 & 0,049 \\
\hline Intensidade da tontura & $-0,004$ & 0,966 \\
\hline Frequência da tontura & 0,300 & $<0,001$ \\
\hline
\end{tabular}

rs = coeficiente de correlação de Spearman 
A avaliação da qualidade de vida tem se tornado fundamental para verificar o impacto integral de doenças e tratamentos sob a perspectiva do indivíduo que sofre a doença (TAKANO et al., 2010). De acordo com a literatura, a tontura é mais frequente no gênero feminino. A casuística do presente estudo caracterizou-se por maioria feminina. Achados semelhantes foram encontrados em outros estudos: $81,8 \%$ por Simoceli et al. (2003), 71,4\% por Paradela, Lourenço e Veras (2005), 68,3\% por Gazzola et al. (2006), 68,3\% por Gazzola et al. (2009), 68,3 \% por Santos et al. (2010), 55,5\% por Berticelli, Macedo e Sleifer (2014), em amostras compostas por idosos com disfunção vestibular crônica. Esses dados podem ser justificados pela maior preocupação das mulheres em procurar atendimento médico em relação aos homens, como também pela associação de alterações vestibulares com alterações metabólicas e hormonais no gênero feminino, o que tornaria a tontura mais frequente nas mulheres (PEDALINI et al., 1999; GAZZOLA et al., 2006; GAZZOLA et al., 2009; SANTOS et al., 2010; BERTICELLI; MACEDO; SLEIFER, 2014).

Indivíduos que apresentam tontura podem reduzir suas atividades de vida diária em virtude do aparecimento dos sintomas durante a realização delas, acarretando prejuízos na qualidade de vida (TAKANO et al., 2010). Os achados do presente estudo evidenciam o impacto negativo da tontura na qualidade de vida dos idosos com esta queixa. Vaz e Oliveira (2007) estudaram o impacto da vertigem na qualidade de vida de pacientes com alteração do equilíbrio corporal e evidenciaram diminuição em todos os itens avaliados pelo SF-36.

A mediana dos escores nos domínios capacidade funcional, limitação por aspectos físicos e por aspectos emocionais foram 55, 0 e 33,3, respectivamente, em uma escala que vai de zero a 100, sendo zero a pior pontuação e 100, a melhor. Não foram encontrados estudos que utilizassem o questionário SF-36 para avaliar a mesma população. Outros estudos, que utilizaram o DHI, Diziness Handcap Inventory, traduzido para o português e validado por Castro (2003), observaram que pacientes com distúrbio do equilíbrio corporal apresentam prejuízo na qualidade de vida em relação aos aspectos físicos, funcionais e emocionais, que compreendem o instrumento (GANANÇA et al., 2004; SANTOS et al., 2010; TAKANO et al., 2010).

A insegurança física provocada pela tontura resulta, muitas vezes, em sintomas psicológicos (YESAVAGE; SHEIK, 1986; PAULINO; PREZOTTO; CALIXTO, 2009). Nos idosos, a ocorrência de sintomas depressivos é um importante problema de saúde, o que pode explicar os baixos escores nos 
domínios limitação por aspectos físicos e limitação por aspectos emocionais encontrados no presente estudo.

A avaliação dos sintomas depressivos evidenciou que $64,1 \%$ da casuística apresentou escores sugestivos de depressão, ou seja, escores acima de cinco pontos. A prevalência de sintomas depressivos encontrada no presente estudo é semelhante à encontrada no estudo de Gazzola et al. (2009), em que mais da metade da população estudada pontuou acima do ponto de corte na GDS e superior à encontrada por Tusa (2002), em que 40\% dos pacientes com tontura apresentaram alterações dessa ordem.

No estudo Paiva e Kuhn (2004), a concomitância de sintomas psicológicos à queixa de tontura foi verificada em 477 (56,38\%) de um total de 846 prontuários de pacientes avaliados em um Ambulatório de Otoneurologia, sendo que, dentre estes, a depressão teve uma prevalência de $12,58 \%$. No estudo de Gurgel et al. (2012), que buscava verificar a associação entre os resultados do exame vestibular e queixa psicológica autorrelatada, verificou-se que 94,7\% dos indivíduos que relataram queixa psicológica referiram, também, queixa de tontura e/ou vertigem, porém, dentre estes, 55,3\% apresentaram resultado normal ao exame vestibular.

Na presente casuística, a prevalência de sintomas depressivos foi semelhante entre homens e mulheres $(p=0,723)$. Esses dados corroboram os achados de Paradela, Lourenço e Veras (2005), que, ao avaliarem indivíduos com mais de 65 anos, por meio da GDS, concluíram que o diagnóstico de transtorno de humor foi semelhante em ambos os gêneros. Em contrapartida, o estudo de Gazzola et al. (2009), ao avaliar idosos com disfunção vestibular crônica por meio da GDS, concluiu que o maior número de sintomas depressivos em idosos está relacionado com o gênero feminino.

Também não foram encontradas diferenças estatisticamente significantes entre homens e mulheres quanto à qualidade de vida avaliada pelos domínios do questionário SF-36 neste estudo. A correlação entre a GDS e o SF-36 apontou que quanto maior o grau da depressão, menor a qualidade de vida em todos os domínios, mostrando que houve relação significativa entre os oito domínios do SF-36 e a presença de sintomas depressivos. O mesmo pode ser observado com relação ao aspecto frequência da tontura, presença de humor deprimido e prejuízos na qualidade de vida. Os idosos com crises frequentes de tontura apresentaram maior pontuação na GDS e pior qualidade de vida do que os idosos que apresentam crises esporádicas, corroborando com o estudo realizado por Hsu et al. (2005), que observaram correlação entre a frequência das crises de tontura e a percepção de incapacidade. 
No presente estudo, a tontura se associou inversamente com todos os domínios do SF-36, exceto no domínio de dor, ou seja, quanto mais frequente a tontura, menor a qualidade de vida. Da mesma forma, em outro estudo, ao aplicar o questionário SF-36 em indivíduos com e sem distúrbio do equilíbrio corporal, os autores encontraram diminuições nas porcentagens totais dos pacientes com tontura quando comparadas com outro grupo, menos no domínio dor, que foi menor no grupo sem comprometimento vestibular (VAZ; OLIVEIRA, 2007).

Esses resultados podem ser atribuídos ao fato de que muitos idosos são portadores de múltiplas patologias crônicas (DUARTE; REGO, 2007), com alta prevalência de doenças provenientes do sistema osteomolecular e conjuntivo (GAZZOLA et al., 2006), que constituem na queixa mais frequente de dor em indivíduos idosos (SADER; ROSSI, 2002).

Ao analisar sintomas depressivos e fatores associados em uma população idosa, os autores encontraram médias estatisticamente maiores de sintomas depressivos em indivíduos mais velhos (GAZALLE et al., 2004). A presente amostra evidenciou associação direta entre a pontuação na GDS e a idade. Semelhantemente, ao avaliar qualidade de vida de idosos com tontura, os autores de um estudo verificaram que pacientes com idade superior a 81 anos apresentaram valores de maior comprometimento ao $D H I$ brasileiro do que os pacientes com inferior faixa etária, inclusive no aspecto emocional avaliado nesse inventário (TAKANO et al., 2010).

Ao associar a variável frequência da tontura e o grau de depressão, as análises mostraram que a maior periodicidade da tontura relacionou-se com maiores pontuações na GDS. Esses achados corroboram outros autores, que encontraram uma correlação entre tontura crônica e piora de sintomas depressivos na amostra estudada (TINETTI; WILLIAMS; GILL, 2000).

Os resultados deste estudo demonstram os prejuízos na qualidade de vida de idosos com tontura e sua forte relação com a ocorrência de sintomas depressivos, ressaltando a necessidade de incluir a aplicação de questionários genéricos de qualidade de vida e de rastreamento de depressão nessa população.

Os achados corroboram dados prévios da literatura que destacam os prejuízos da tontura na qualidade de vida de indivíduos idosos, bem como a presença de sintomas depressivos concomitantes a essa queixa nessa população, chamando atenção para a necessidade de uma abordagem multidisciplinar na avaliação e no manejo terapêutico desses pacientes. 
Esta pesquisa permitiu concluir que idosos com queixa de tontura apresentam prejuízos significativos na qualidade de vida. Na amostra estudada, a prevalência de sintomas depressivos foi significativa, não havendo diferenças significantes entre homens e mulheres.

Houve correlação entre a presença de sintomas depressivos e prejuízos na qualidade de vida de idosos com tontura, como também houve correlação direta entre o grau de depressão com a idade e com a frequência de tontura, evidenciando que quanto maior a idade e a frequência da tontura, maior é o grau de depressão.

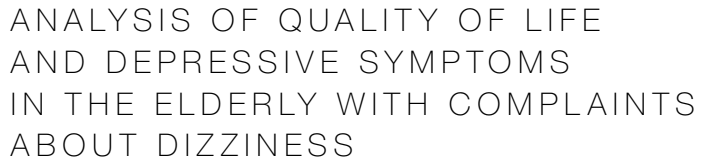

abstract

Objective: to analyze the quality of life and depressive symptoms in the elderly with dizziness. Methodology: this is a cross-sectional, individual, observational and contemporary study. We analyzed the responses of History, SF-36 and Geriatric Depression Scale (GDS) of 153 elderly patients with dizziness. Results: $64.1 \%$ of the seniors scored above the cutoff on the Geriatric Depression Scale, and the levels were similar between men and women. There was an inverse correlation between the GDS score and all domains of the SF-36. Thus, the higher the score on the GDS, the lower was the quality of life in all areas. The frequency of dizziness was inversely associated with virtually all domains of the SF-36, except for pain, that is, the more frequent dizziness, lower was the quality of life. There was a direct association between the GDS score with age and frequency of dizziness. Therefore, the higher the age and frequency of dizziness, the greater was the degree of depression. Conclusion: the elderly with dizziness have significant losses in quality of life. The prevalence of depressive symptoms was significant in the sample. There was an association between depressive symptoms and impaired quality of life.These findings corroborate data from the literature that highlight the damage the patient's quality of life of elderly individuals as well as the concomitant presence of depressive symptoms in those subjects 
to this complaint, calling attention to the need a multidisciplinary

keywords

Dizziness. Aged. Quality of Life. Depression.

referências

BERTICELLI, Amanda Zanatta; MACEDO, Luciana Baú; SLEIFER, Pricila. Effectiveness of Vestibular Rehabilitation in Elderly Subjects with Dizziness. In: CONGRESSO DA FORL, 13., 2014, Goiânia. Resumos...Goiânia: Fundação Otorrinolaringologia, 2014. p. 35.

BERTOLUCCI, Paulo Henrique Ferreira et al. Proposta de padronização do Mini-Exame do Estado Mental (MEEM): estudo piloto cooperativo. Arquivos de Neuro-Psiquiatria, São Paulo, v. 52, p. 225, 1994.

BITTAR, Roseli Saraiva Moreira; GANANÇA, Fernando Freitas. Decifrando a tontura. 2. ed. São Paulo: Segmento Farma, 2010. p. 9-13.

BRASIL. Ministério da Saúde. Portaria n. ${ }^{\circ} 1395$, de 09 de dezembro de 1999. Política Nacional do Idoso. Diário Oficial [da] República Federativa do Brasil, Poder Executivo, Brasília, DF, 9 dez. 1999

BRUCKI, Sonia Maria Dozzi et al. Sugestões para o uso do Mini-Exame do Estado Mental no Brasil. Arquivos de Neuro-Psiquiatria, São Paulo, v. 61, n. 3, p. 777-781, 2003.

CAMPOLINA, Alessandro Gonçalves; CICONELLI, Rozana Mesquita. O SF-36 e o desenvolvimento de novas medidas de avaliação de qualidade de vida. Acta Reumatológica Portuguesa, Lisboa, v. 33, n. 2, p. 127-133, 2008.

CASTRO, Ana Sílvia Oliveira de. Dizziness Handicap Inventory: adaptação cultural para o português brasileiro, aplicação, reprodutibilidade e comparação com os resultados à vestibulometria. 2003. 59 f. Dissertação (Mestrado em Ciências da Reabilitação Neuro-motora) - Universidade Bandeirante de São Paulo, São Paulo, 2003.

CICONELLI, Rozana Mesquita. Tradução para o português e validação do questionário genérico de avaliação da qualidade de vida "Medical Outcomes Study 36 -item ShortForm Health Survey (SF-36)". 1997. 145 f. Tese (Doutorado em Medicina) - Escola Paulista de Medicina, Universidade de São Paulo, São Paulo, 1997.

DUARTE, Meirelayne Borges; REGO, Marco Antônio Vasconcelos. Comorbidade entre depressão e doenças clínicas em um ambulatório de geriatria. Cadernos de Saúde Pública, Rio de Janeiro, v. 23, n. 3, p. 691-700, Mar. 2007.

FELIPE, Lilian et al. Presbivertigem como causa de tontura no idoso. Pró-Fono Revista de Atualização Científica, Barueri, v. 20, n. 2, p. 99-104, abr./jun. 2008.

FIGUEIREDO, Anelise et al. Depressão no idoso. In: TERRA, Newton Luiz; DORNELLES, Beatriz (Org.). (2002). Envelhecimento bem-sucedido. 2. ed. Porto Alegre: EDIPUCRS, 2003. p. 149-60

FOLSTEIN, Marshal F.; FOLSTEIN, Susan E.; McHUGH, Paul R. Mini-mental state: a practical method for grading the cognitive state of patients for the clinician. Journal of Psychiatric Research, Oxford, v. 12, p. 189-198, 1975.

GANANÇA, Maurício Malavasi, CAOVILLA, Heloísa Helena. Desequilíbrio e reequilíbrio. In: GANANÇA, Maurício Malavasi. Vertigem tem cura? São Paulo: Lemos Editorial, 1998. p. 13-19. 
GANANÇA, Fernando Freitas et al. Interferência da tontura na qualidade de vida de pacientes com síndrome vestibular periférica. Revista Brasileira de Otorrinolaringologia, São Paulo, v. 70, n. 1, p. 94-101, jan./fev. 2004

GAZALLE, Fernando Kratz et al. Sintomas depressivos e fatores associados em população idosa no Sul do Brasil. Revista de Saúde Pública, São Paulo, v. 38, n. 3, p. 365-371, jun. 2004.

GAZZOLA, Juliana Maria et al. Factors relating to depressive symptoms among elderly people with chronic vestibular dysfunction. Arquivos de Neuro-Psiquiatria, São Paulo, v. 67, n. 2B, p. 416-422, jun. 2009.

GAZZOLA, Juliana Maria et al. Caracterização clínica de idosos com disfunção vestibular crônica. Revista Brasileira de Otorrinolaringologia, São Paulo, v. 72, n. 4, p. 515-522, jul./ago. 2006.

GURGEL, Léia Gonçalves et al. Correlation between vestibular test results and selfreported psychological complaints of patients with vestibular symptoms. Brazilian Journal of Otorhinolaryngology, São Paulo, v. 78, n. 1, p. 62-67, jan./fev. 2012.

HONG, Seok Min et al. Analysis of psychological distress after management of dizziness in old patients: multicenter study. European Archives of Oto-Rhino-Laryngology, v. 269, n. 1, p. 39-43, Jan. 2012

HSU, Li-Chi et al. Quality of life in elderly patients with dizziness: analysis of the ShortForm Health Survey in 197 patients. Acta Oto-Laryngologica, Stockholm, v. 125, n. 1, p. 55-59, Jan. 2005

IÓRIO, Maria Cecília Martinelli. Avaliação de Resultados - Estudos de Qualidade de Vida. In: BEVILACQUA, Maria Cecília et al. Tratado de Audiologia. 1. ed. São Paulo: Santos, 2011. p. 389-403.

MANTELLO, Erika Barioni et al. Efeito da reabilitação vestibular sobre a qualidade de vida de idosos labirintopatas. Revista Brasileira de Otorrinolaringologia, São Paulo, v. 74, n. 2, p. 172-180, mar./abr. 2008.

PAIVA, Angela Daou; KUHN, Ana Maria Baccari. Sintomas psicológicos concomitantes à queixa de vertigem em 846 prontuários de pacientes otoneurológicos do Ambulatório de Otoneurologia da Universidade Federal de São Paulo - Escola Paulista de Medicina. Revista Brasileira de Otorrinolaringologia, São Paulo, v. 70, n. 4, p. 512-515, jul./ago. 2004.

PARADELA, Emylucy Martins Paiva; LOURENÇO, Roberto Alves; VERAS, Renato Peixoto. Validação da escala de depressão geriátrica em um ambulatório geral. Revista de Saúde Pública, São Paulo, v. 39, n. 6, p. 918-923, dez. 2005

PAULINO, Célia Aparecida; PREZOTTO, Andrea Oriani; CALIXTO, Rosilene Farias. Associação entre estresse, depressão e tontura: uma breve revisão. Revista Equilíbrio Corporal e Saúde, São Paulo, v. 1, n. 1, p. 33-45, 2009.

PEDALINI, Maria Elisabete Bovino et al. Reabilitação vestibular como tratamento de tontura: experiência com 116 casos. International Archives of Otorhinolaryngology, São Paulo, v. 3, n. 2, p. 74-78, abr./jun. 1999.

RUWER, Sheelen Larissa; ROSSI, Angela Garcia; SIMON, Larissa Fortunato. Equilíbrio no idoso. Revista Brasileira de Otorrinolaringologia, São Paulo, v. 71, n. 3, p. 298-303, maio/jun. 2005.

SADER, Cristina S.; ROSSI, Edison. O envelhecimento do sistema osteoarticular. In: FREITAS, Elizabete Viana de; PY, Ligia (Ed.). Tratado de Geriatria e Gerontologia. Rio de Janeiro: Guanabara Koogan, 2002. p. 832-838.

SANTOS, Erika Maria dos et al. Impacto da tontura na qualidade de vida de idosos com vestibulopatia crônica. Pró-Fono Revista de Atualização Científica, Barueri, v. 22, n. 4, p. 427-432, out./dez. 2010 
SIMOCELI, Lucinda et al. Perfil diagnóstico do idoso portador de desequilíbrio corporal: resultados preliminares. Revista Brasileira de Otorrinolaringologia, São Paulo, v. 69, n. 6, p. 772-777, nov./dez. 2003.

TAKANO, Nancy Akemi et al. Quality of life in elderly with dizziness. Brazilian Journal of Otorhinolaryngology, São Paulo, v. 76, n. 6, p.769-775, nov./dez. 2010.

TINETTI, Mary Elizabeth; WILLIAMS, Christianna; GILL, Thomas M. Dizziness among older adults: a possible geriatric syndrome. Annals of Internal Medicine, Philadelphia, PA, v. 132, n. 5, p. 337-344, Mar. 2000.

TUSA, Ronald. Problemas psicológicos e o paciente com vertigem. In: HERDMAN, Susan. Reabilitação Vestibular. Rio de Janeiro: Manole, 2002. p. 312-315.

VAZ, Aretha Cintra; OLIVEIRA, Ana Paula Rocha de. Impacto da vertigem na qualidade de vida em pacientes com disfunção vestibular. Fisioterapia em Movimento, Curitiba, v. 20, n. 1, p. 71-76, jan./mar. 2007.

WARE, John E. et al. SF-36 health survey: manual and interpretation guide. Boston, MA: Health Assessment Lab, 1993.

WORLD HEALTH ORGANIZATION (WHO). International Classification of Impairments, Disabilities and Handicaps: A Manual of Classification Relating to the Consequences of Disease. Geneva: WHO, 1980

YESAVAGE, Jerome A.; SHEIKH, Javaid I. Geriatric depression scale (GDS): recent evidence and development of a shorter version. Clinical Gerontologist, New York, v. 5, n. $1-2$, p. $165-173,1986$

Recebido: 19/06/2014

Aceite Final: 17/08/2015 
